\title{
Computational modeling of thermoluminescence glow curves of zinc borate crystals
}

Nil Kucuk and Ilker Kucuk

${ }^{*}$ Correspondence:

nilkoc@uludag.edu.tr

Physics Department, Faculty of Arts

and Sciences, Uludag University,

Gorukle Campus, Bursa, 16059,

Turkey

\begin{abstract}
This work presents a new approach based on multilayered perceptrons (MLPs) to compute the thermoluminescence glow curves in undoped and La-doped zinc borate crystals. The MLP has been trained by a genetic algorithm (GA) and a Levenberg-Marquardt learning algorithm. The results obtained using the MLP models were tested with untrained experimental glow curve data. The comparison has shown that the proposed model can predict more accurately and easily the thermoluminescence glow curves.
\end{abstract}

PACS Codes: $78.60 . \mathrm{Kn} ; 87.53 . \mathrm{Bn}$

Keywords: dosimetric materials; thermoluminescence glow curve; thermally stimulated luminescence; modeling

\section{Introduction}

Controlled measurement of the emitted light (glow curve) of a dosimetric material is normally used for determination of the radiation dose absorbed by that material [1]. To understand the behavior of the dosimetric material, it is necessary to analyze the glow curve and evaluate the trap parameters [2]. Glow curve analysis is widely used for dosimetric studies [3-9].

Most insulating or semiconducting materials generally exhibit thermoluminescence (TL) glow curves with one or more peaks when the charge carriers are released. The peaks observed on this curve correspond to the ionization of traps at various energy levels of the dosimetric material. These traps are characterized by certain physical parameters. In order to obtain these important physical parameters, one needs to fit the glow curve data to a TL model that best describes the TL intensity as a function of these parameters. Thus, the developed TL model is very useful for getting detailed information on kinetic parameters of trapping centers in the dosimetric material. Kinetic parameters are activation energy $(E)$, frequency factor $(s)$ and the order of kinetics $(b)$. $E$ and $s$ have been determined by variable heating rate (VHR), initial rise (IR), isothermal decay (ID), and peak shape (PS) methods. Also, $b$ has been generally calculated by PS methods from the peak shape parameters.

The generalization ability, real-time operation, and ease of application have made artificial neural network (ANN) quite popular in the last years [10]. ANNs have been applied in many areas because of these features [11-16]. The ANN software available today pro-

\section{空 Springer}

(c) 2013 Kucuk and Kucuk; licensee Springer. This is an Open Access article distributed under the terms of the Creative Commons Attribution License (http://creativecommons.org/licenses/by/2.0), which permits unrestricted use, distribution, and reproduction in any medium, provided the original work is properly cited. 
vides many neural network architectures and learning algorithms, to be applied to specific problems.

The purpose of the present work is to model the thermoluminescence glow curves of trapping centers in undoped and La-doped zinc borate crystals using GA, ANNs, and experimental data. The proposed model is not time-consuming and can more accurately and easily predict the thermoluminescence glow curves of zinc borate crystals.

\section{Experimental details}

Powder samples of undoped and La-doped $\mathrm{ZnB}_{2} \mathrm{O}_{4}$ at 1\%, 2\%, 3\%, 4\%, 5\%, and $10 \%$ (by weight) were prepared by the nitric acid method. This method is relatively easy and cheap. $\mathrm{ZnB}_{2} \mathrm{O}_{4}$ powder samples were synthesized using zinc oxide $(\mathrm{ZnO}$, with a minimum purity of $99.99 \%)$ and boric acid $\left(\mathrm{H}_{3} \mathrm{BO}_{3}\right.$, with a purity of $\left.99.99 \%\right)$. Appropriate amounts of $\mathrm{ZnO}$ and $\mathrm{H}_{3} \mathrm{BO}_{3}$ powders were separately weighted to prepare zinc borate. Starting materials were mixed, while heating at $80^{\circ} \mathrm{C}$, in $1 \mathrm{M}$ nitric acid solution $\left(\mathrm{HNO}_{3}\right.$, standard solution) by using a magnetic stirrer. All the experiments were carried out in a glass beaker of $250 \mathrm{ml}$ volume. For $5 \mathrm{~g}$ of initial powders, $80 \mathrm{ml}$ acid was used. During this process, all oxides and boric acid were converted into the metallic nitrates [i.e., $\mathrm{Zn}\left(\mathrm{NO}_{3}\right)_{2}, \mathrm{~B}\left(\mathrm{NO}_{3}\right)_{3}$ and $\left.\mathrm{La}\left(\mathrm{NO}_{3}\right)_{3}\right]$. La-doped $\mathrm{ZnB}_{2} \mathrm{O}_{4}$ powder samples were also prepared in a similar manner by taking the starting material in stoichiometric ratio and adding $\mathrm{La}_{2} \mathrm{O}_{3}$ to the mixture [17].

Mixing was continued until a dry precursor was obtained. The precursor was ground in an agate mortar for about $15 \mathrm{~min}$. Then, it was calcined at $450^{\circ} \mathrm{C}$ for $5 \mathrm{~h}$ to remove possible organic compounds. Gasses such as $\mathrm{NO}$ and $\mathrm{NO}_{2}$ were also released up to this temperature, and metallic nitrates were converted into the oxides again [18]. Finally, the precursor was pelletized under the pressure of 3 ton before annealing at temperatures from $700^{\circ} \mathrm{C}$ to $900^{\circ} \mathrm{C}$ for $2 \mathrm{~h}$ which led to the formation of zinc borates. After annealing, the powder samples were cooled to room temperature and triturated in an agate mortar [17].

The structure analysis of all the samples and the effect of doping on the structure of undoped $\mathrm{ZnB}_{2} \mathrm{O}_{4}$ powder sample were studied by X-ray powder diffraction. The X-ray diffraction (XRD) measurements were taken at the interval of Bragg angle $2 \theta$ from $\left(10^{\circ}<\right.$ $2 \theta<90^{\circ}$ ), using a Rigaku Ultima IV X-ray diffractometer at $40 \mathrm{kV}$ at $3 \mathrm{deg} \cdot \mathrm{min}^{-1}$ and $30 \mathrm{~mA}$ with $\mathrm{Cu}-\mathrm{K}_{\alpha}(\lambda=1.5405 \AA)$ radiation [17].

The TL glow curves of undoped and various La-doped $\mathrm{ZnB}_{2} \mathrm{O}_{4}$ powder samples were recorded with Risø TL/OSL DA-20 reader using Corning 7/59 and Schott BG/39 optical filters in nitrogen atmosphere. The measurements were carried out on $10 \mathrm{mg}$ samples. During measurements of all the powder samples, pre-heating process up to $140^{\circ} \mathrm{C}$ for the heating rate of $2^{\circ} \mathrm{C} / \mathrm{s}$ and the reading process up to $450^{\circ} \mathrm{C}$ for the heating rate of $5^{\circ} \mathrm{C} / \mathrm{s}$ were used. TL intensity for each glow peak was calculated by taking the area under dosimetric peak. The dose responses of all the powder samples exposed to ${ }^{90} \mathrm{Sr}$ beta radiation $(40 \mathrm{mCi})$ were obtained in the dose range from $143 \mathrm{mGy}$ to 60 Gy [17].

\section{Multilayered perceptron neural network}

There are many types of neural networks for various applications available in the literature. Multilayered perceptrons (MLPs) are feed-forward networks and universal approximators. MLPs are the simplest and therefore most commonly used neural network ar- 
chitectures [10]. In this paper, they have been adapted to the computation of the thermoluminescence glow curves of trapping centers in undoped and La-doped zinc borate crystals.

The MLP used in this work is trained with the GA. An MLP consists of three layers: an input layer, an output layer, and an intermediate or hidden layer. Processing elements (PE) or neurons in the input layer only act as buffers for distributing the input signals $x_{i}$ ( $i$ shows the $i$ th input PE) to PEs in the hidden layer. Each $\mathrm{PE}_{j}$ ( $j$ shows the $j$ th $\mathrm{PE}$ in the hidden layer and output layers) in the hidden layer sums up its input signals $x_{i}$ after weighting with the values of the respective connections $w_{j i}$ from the input layer and computes its output $y_{j}$ as a function $f$ of the sum,

$$
y_{j}=f\left(\sum w_{j i} x_{j}\right)
$$

$f$ can be a simple threshold function, a sigmoid or hyperbolic tangent function. The output of PEs in the output layer is computed similarly. Training a network consists of adjusting its weights using a training algorithm. The training algorithms adopted in this study optimize the weights by attempting to minimize the sum of squared differences between the desired and the actual values of the output neurons [10], namely

$$
E=\frac{1}{2} \sum_{j}\left(y_{d j}-y_{j}\right)^{2}
$$

where $y_{d j}$ is the desired value of an output neuron $j$ and $y_{j}$ is the actual output of that neuron. Each weight $w_{j i}$ is adjusted by adding an increment $\Delta w_{j i}$ to it. $\Delta w_{j i}$ is selected to reduce $E$ as rapidly as possible. The adjustment is carried out over several training iterations until a satisfactorily small value of $E$ is obtained or a given number of iterations are reached. The computed $\Delta w_{j i}$ depends on the training algorithm adopted. There are a number of training algorithms used to train a MLP, and a frequently used one is called the back propagation (BP) training algorithm [10]. The BP algorithm, which is based on searching an error surface using gradient descent for points with minimum error, is relatively easy to implement. However, the BP algorithm has some problems for many applications [19]. The algorithm is not guaranteed to find the global minimum of the error function since gradient descent may get stuck in local minima, where it may remain indefinitely. In addition to this, long training sessions are often required in order to find an acceptable weight solution because of the well-known difficulties inherent in gradient descent optimization [19].

In this work target TL intensity $(I)$ for the MLP has been determined by a GA. The computation process is carried out with a set of thermally stimulated luminescence (TSL) measurements.

\section{Determination of target TL intensity with a genetic algorithm}

The GA method is based on a computer simulation of biological evolution and initially works with a randomly generated population with several variables to be estimated [20]. The population size is usually related to the problem under consideration and can be determined by a number of variables. Each member or individual of the population is usually called a chromosome or a string consisting of genes or bits, and encoded into one variable 
(I) for this work. A new population is built up by selecting individuals among members of the initial population according to their fitnesses through a fundamental genetic process of selection criterion based on the roulette wheel. The fitness function $(f f)$ is calculated by

$$
f f=\frac{1}{\sum_{k=1}^{n}\left(I_{t-d, k}-I_{t-c, k}\right)^{2}},
$$

where $n$ is the population size, $I_{t-d}$ and $I_{t-c}$ are the desired and computed TL intensities, respectively.

Fitness value for each string was calculated using the fitness function, and hence new members were chosen for reproduction according to their fitness based on the specified selection criterion. Thus, the fittest had a greater chance to be selected for next population. Once the reproduction was completed, a crossover operation was implemented by simply exchanging bits between two randomly selected members in the population. The final genetic process was mutation that randomly changes a particular bit in a particular string, that is, a zero bit may change to a one or vice versa.

\section{Results and discussion}

The proposed technique involves training an MLP to compute the thermoluminescence glow curves when the values of sintering temperature $\left(T_{\mathrm{s}}\right)$, La-dope amount $\left(D_{\mathrm{La}}\right)$, dose $\left(D_{\mathrm{Gy}}\right)$, and temperature $(T)$ are given. The ranges of training data set were $900^{\circ} \mathrm{C} \leq T_{\mathrm{s}} \leq$ $950^{\circ} \mathrm{C}$ at two points, $0 \leq D_{\mathrm{La}} \leq 10$ at seven points, $0 \mathrm{~Gy} \leq D_{\mathrm{Gy}} \leq 60$ Gy at seven points, $5^{\circ} \mathrm{C} \leq T \leq 450^{\circ} \mathrm{C}$ at 250 points. The TL intensity configuration to be modeled by the neural network is shown in Figure 1. Training an MLP using the GA to compute $I$ involves presenting them with different sets $\left(T_{\mathrm{s}}, D_{\mathrm{La}}, D_{\mathrm{Gy}}\right.$, and $\left.T\right)$ sequentially and/or randomly and corresponding calculated values $I$. Differences between the target output $(I)$ and the actual outputs $\left(I_{- \text {ANN }}\right)$ of the MLP are calculated through the network to adapt its weights using Eqs. (1)-(3). The adaptation is carried out after the presentation of each set $\left(T_{\mathrm{s}}, D_{\mathrm{La}}\right.$, $D_{\mathrm{Gy}}$, and $T$ ) until the calculation accuracy of the network is found satisfactory according to some criterion (for example, when the errors between $I$ and $I_{- \text {ANN }}$ for all the training set fall below a given threshold) or the maximum allowable number of epochs (the time periods that encompass all the iterations performed after all the patterns are presented to the network) is reached.

Furthermore, in order to understand the MLPs prediction accuracy and generalization capacity, the networks were also trained with the training set, cross-validation set and checked with test data. The network memorizes the training set and does not generalize well when the network is trained too much [19]. The training holds the key to an accurate solution, so the criterion to stop training must be very well described. Cross-validation is a highly recommended criterion for stopping the training of a network. When the error in the cross-validation increases, the training should be stopped. A practical way to find a point of better generalization is to use a small percentage (around 10\%) of the training set for cross-validation. To obtain a better generalization of the networks presented in this work, 1,570 of training data, which were selected randomly, were used as a cross-validation set.

In total 15,750 data sets were used in training and test phases. 500 data sets were used to test the network. For the validation, untrained experimental data were used to test the 


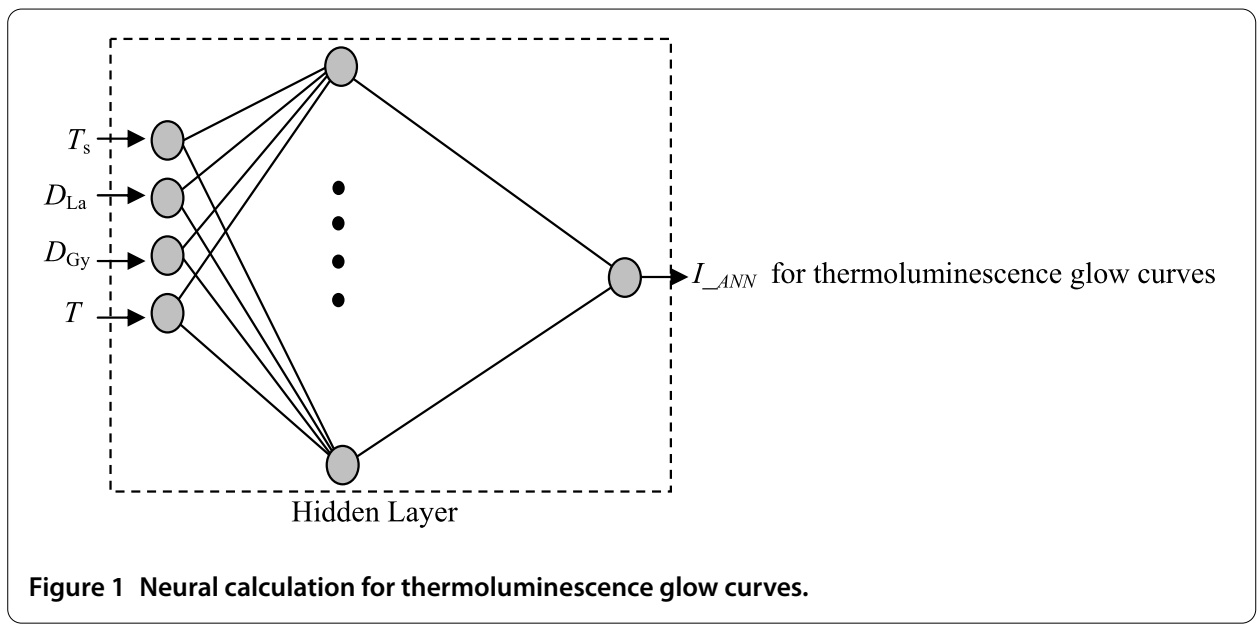

neural model as well. The number of hidden layers and neurons in each layer was determined through trial and error to be optimal including different transfer functions as hyperbolic tangent, sigmoid and hybrid. After trials, a better result was obtained from a three-layered network. In this network, the hyperbolic tangent function is used in the hidden layer, and sigmoid function is used in the output layer. The number of epochs was 1,000 for training, and the most suitable network configuration found was $4 \times 24 \times 1$. It means that the number of neurons was 24 for the hidden layer.

In the proposed model, 63 thermoluminescence glow curves in undoped and La-doped zinc borate were used. The estimates of $I$ were found to be in a range from 1,553,559 to -5 by use of measured TL intensity results in the zinc borate crystals at temperatures varying from 41.64 to $450^{\circ} \mathrm{C}$. The correlation coefficient for the trained data was found to be 0.9982 .

All tested thermoluminescence glow curves of zinc borate crystals in the range of training data have high correlation coefficients. Figure 2 shows variation with temperature of TL intensity obtained from the neural network model and experimental data for $\mathrm{ZnB}_{2} \mathrm{O}_{4}: 0.03 \mathrm{La}$ powder sample. The values of TL intensity achieved from the proposed model are in $99 \%$ agreement with the experimental values of the TL intensity. The model was assessed for $\mathrm{ZnB}_{2} \mathrm{O}_{4}$ powder sample exposed to $60 \mathrm{~Gy}$ which is outside the range of the training data. The variation of the TL intensity with temperature for $\mathrm{ZnB}_{2} \mathrm{O}_{4}$ powder sample given in Figure 3 also shows good correlation between measured and predicted results.

The proposed method has some inherent limitations which make it not a general solution. The trained neural network is based on a specific set of zinc borates. The trained network can be only valid for the same doped and undoped zinc borates. For different dosimetric materials, a series of experiments would have to be performed again to obtain input data for the proposed ANN training. If the ANN input data could include the TL glow curve information of new materials, many more experimental data would have to be done to meet the accuracy requirements for a more general solution. However, since the neural model presented in this work has accuracy and requires no tremendous computational efforts and less background information about the thermoluminescence and dosimeter, it can be very useful for the layered single crystals. This model capable of more 


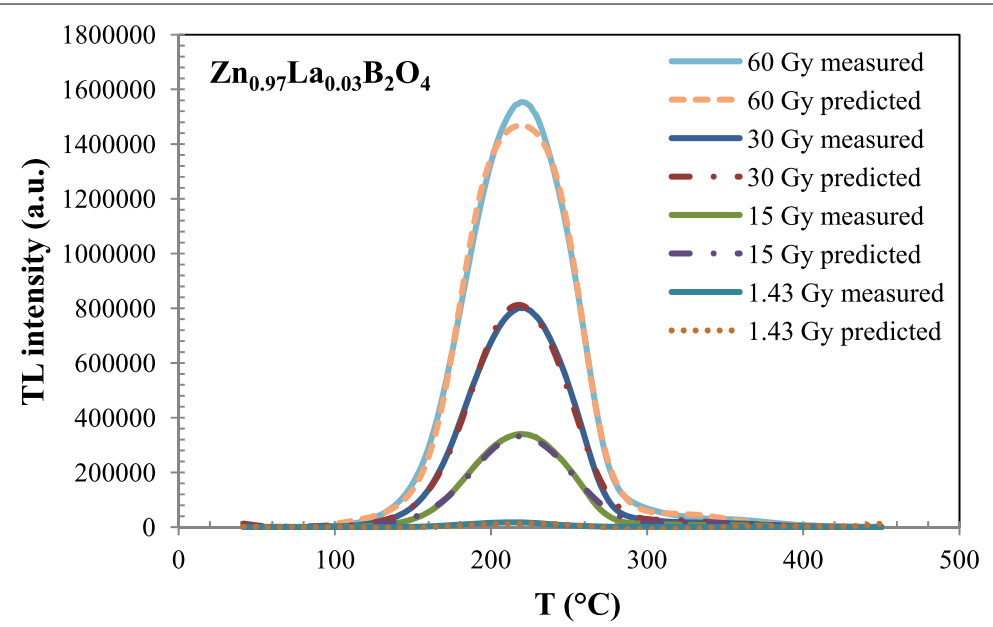

Figure 2 The variation of predicted and measured TL intensity with temperature for $\mathrm{ZnB}_{2} \mathrm{O}_{4}: 0.03 \mathrm{La}$ powder sample exposed to different doses.

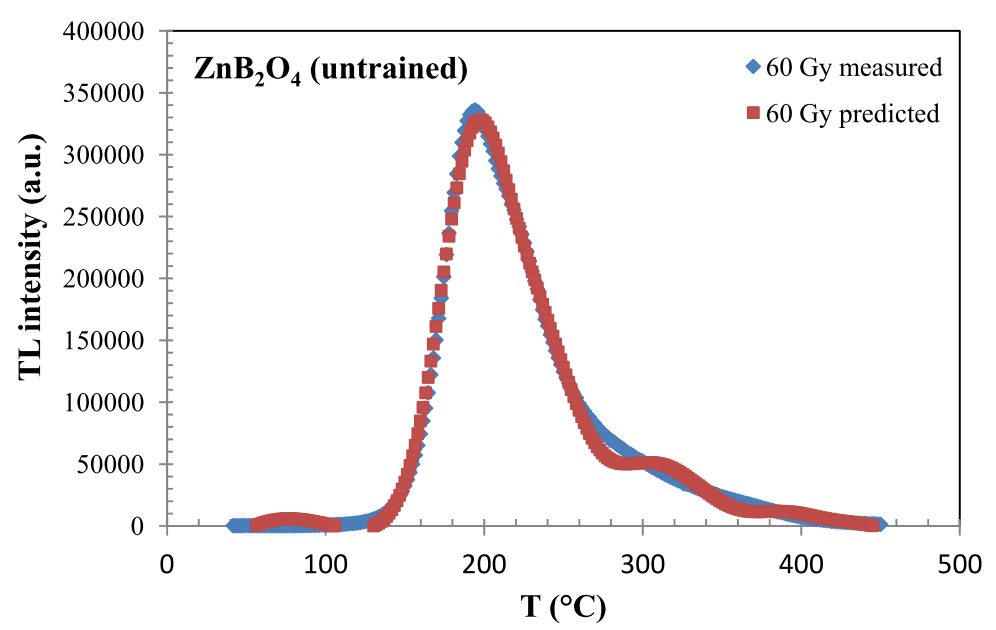

Figure 3 Comparison of predicted and measured TL glow curves for $\mathrm{ZnB}_{2} \mathrm{O}_{4}$ powder sample exposed to $60 \mathrm{~Gy}$.

accurately predicting thermoluminescence glow curves in La doped zinc borates is also very useful to a researcher working in this field.

\section{Conclusions}

The predicted results from the ANN approach sound very satisfactory and in agreement with experimental results concerning the thermoluminescence glow curves in undoped and La doped zinc borates. The model is fast and allows the application of standard learning algorithms to the neural network.

\section{Competing interests}

The authors declare that they have no competing interests.

\section{Authors' contributions}

All authors carried out the proof. All authors conceived of the study, and participated in its design and coordination. All authors read and approved the final manuscript. 


\section{Acknowledgements}

Dedicated to Professor Hari M Srivastava.

This work was supported by the Commission of Scientific Research Projects of Uludag University, Project number UAP(F)-2011/74

Received: 25 January 2013 Accepted: 11 March 2013 Published: 29 March 2013

\section{References}

1. Abd El-Hafez, Al, Yasin, MN, Sadek, AM: GCAFIT - a new tool for glow curve analysis in thermoluminescence nanodosimetry. Nucl. Instrum. Methods Phys. Res. A 637, 158-163 (2011)

2. Furetta, C, Kitis, G, Kuo, CH: Kinetics parameters of CVD diamond by computerized glow-curve deconvolution (CGCD). Nucl. Instrum. Methods Phys. Res. B 160, 65-72 (2000)

3. Bos, AJJ, Piters, TM, Gomez Ros, JM, Delgado, A: GLOCANIN, an Intercomparision of Glow Curve Analysis Computer Programs. IRI-CIEMAT Report 131-93-005, IRI, Delft (1993)

4. Strauss, L, Hajek, M: Review of methods for the determination of the kinetic parameters of thermoluminescence dosimeters. Project thesis by Strauss, L, Atomic Institute of Austrian Universities, Vienna, Austria. www.ati.ac.at (2007)

5. Horowitz, YS, Yossian, D: Computerized glow curve deconvolution: application to thermoluminescence dosimetry. Radiat. Prot. Dosim. 60, 1-114 (1995)

6. Gambarini, G, Sinha Roy, M: Dependence of TLD thermoluminescence yield on absorbed dose in a thermal neutron field. Appl. Radiat. Isot. 48, 1467-1475 (1997)

7. Hajek, M, Berger, T, Bergmann, R, Vana, N, Uchihori, Y, Yasuda, N, Kitamura, H: LET dependence of thermoluminescent efficiency and peak height ratio of $\mathrm{CaF}_{2}:$ Tm. Radiat. Meas. 4, 1135-1139 (2008)

8. Furetta, C, Kuo, CH, Weng, PS: Fading prediction in thermoluminescent materials using computerised glow curve deconvolution (CGCD). Nucl. Instrum. Methods Phys. Res. A 423, 183-189 (1999)

9. Ratovonjanhary, AJF, Raboanary, R, Andriambolona, R, Goeksu, HY: Quartz glow-peaks lifetime analysis. TI glow-curve deconvolution functions for first order of kinetic compared to initial rise method. In: Proceedings of the HEPMAD'04 Conference, Madagascar, 27 September-01 October (2004)

10. Haykin, S: Neural Networks: a Comprehensive Foundation. Macmillan Co., New York (1994)

11. Kucuk, I: Prediction of hysteresis loop in magnetic cores using neural network and genetic algorithm. J. Magn. Magn. Mater. 305, 423-427 (2006)

12. Kucuk, I: Prediction of hysteresis loop in magnetic cores using neural network and genetic algorithm. J. Magn. Magn. Mater. 307, 53-61 (2006)

13. Kucuk, N: Modeling of gamma-ray energy absorption buildup factors using neural network. Ann. Nucl. Energy 35 1787-1790 (2008)

14. Hayajneh, MT, Hassan, AM, Mayyas, AT: Artificial neural network modeling of the drilling process of self-lubricated aluminum/alumina/graphite hybrid composites synthesized by powder metallurgy technique. J. Alloys Compd. 478 , 559-565 (2009)

15. Hayajneh, MT, Hassan, AM, Alrashdan, A, Mayyas, AT: Prediction of tribological behavior of aluminum-copper based composite using artificial neural network. J. Alloys Compd. 470, 584-588 (2009)

16. Mirzadeh, $\mathrm{H}, \mathrm{Najafizadeh,} \mathrm{A}$ : ANN modeling of strain-induced martensite and its applications in metastable austenitic stainless steels. J. Alloys Compd. 476, 352-355 (2009)

17. Kucuk, N, Kucuk, I, Cakir, M, Keles, SK: Synthesis, thermoluminescence and dosimetric properties of La-doped zinc borates. J. Lumin. 139, 84-90 (2013)

18. Sözeri, $\mathrm{H}$ : Simple recipe to synthesize single-domain $\mathrm{BaFe}_{12} \mathrm{O}_{19}$ with high saturation magnetization. J. Magn. Magn. Mater. 321, 2717-2722 (2009)

19. Ubeyli, ED, Guler, I: Multilayer perceptron neural networks to compute a quasistatic parameters of asymmetric coplanar waveguides. Neurocomputing 6(2), 349-365 (2004)

20. Goldberg, DE: Genetic Algorithms in Search, Optimization and Machine Learning. Addison-Wesley, Boston (1989)

doi:10.1186/1029-242X-2013-136

Cite this article as: Kucuk and Kucuk: Computational modeling of thermoluminescence glow curves of zinc borate crystals. Journal of Inequalities and Applications 2013 2013:136.

\section{Submit your manuscript to a SpringerOpen ${ }^{\ominus}$ journal and benefit from:}

- Convenient online submission

- Rigorous peer review

- Immediate publication on acceptance

- Open access: articles freely available online

- High visibility within the field

- Retaining the copyright to your article 